\title{
Searches for dark matter and extra dimensions with the ATLAS detector
}

\author{
Michael Kruskal ${ }^{1, a}$, on behalf of the ATLAS Collaboration \\ ${ }^{1}$ Boston University
}

\begin{abstract}
Different approaches to finding evidence for dark matter at the LHC are presented. These include searches for events with large missing transverse momentum and a single jet, photon or $W / Z$ boson. Studies sensitive to the presence of extra spatial dimensions are also described, such as classical or quantum black holes and other non-resonant phenomena. Results are presented from the $\sqrt{s}=8 \mathrm{TeV}$ data taking period.
\end{abstract}

\section{Dark Matter Searches}

\subsection{Background}

Over the last 80 years, an abundance of cosmological evidence of dark matter has been observed. Some of the strongest examples can be found in [1][2][3][4][5][6]. Despite all of these observations, very little is known about what this invisible substance is made of. The only properties that have been gathered from the cosmological data are that it is stable, cold, abundant, and interacts very weakly with ordinary baryonic matter. So far, no non-gravitational interactions of dark matter have been found.

The currently favored description of dark matter is the Weakly Interacting Massive Particle (WIMP) model. The reason for this stems from two observations. The first is that the predicted relic abundance of a particle with weak scale interactions and a weak scale mass would be very close to the observed abundance of dark matter. The second is that most theories beyond the Standard Model (SM) predict the existence of various WIMPs, many of which could be stable. There are alternative models for dark matter, but searches for them are typically very different from the WIMP searches presented here.

There are 3 different ways to search for WIMP dark matter (DM), corresponding to rotations of a feynman diagram coupling dark matter to baryonic matter. The first of these, known as indirect detection, looks for astronomical WIMP-WIMP annihilations that produce visible SM particles [7][8][9][10]. The second, direct detection, looks for local dark matter particles recoiling off atomic nuclei. Direct detection searches can be sensitive to spin-independent interactions [11][12][13][14][15] and spin-dependent interactions [16][17][18], depending on the nuclei used. The final search method, which will be discussed in detail here, is the collider search. Experiments such as ATLAS [19] attempt to observe the production of WIMPs via collisions of Standard Model particles.

\footnotetext{
a e-mail: mkruskal@bu.edu
} 
ATLAS is located on the LHC [20] ring, where protons were collided at a center of mass energy of 8 $\mathrm{TeV}$ in 2012 and a total integrated luminosity of $20.3 \mathrm{fb}^{-1}$ was recorded.

In general, it is very difficult to compare results from all of these different search methods without choosing a specific model. Model-dependent analyses are very limited though, because of the vast number of models beyond the SM that have been formulated. The approach taken by most ATLAS WIMP searches is to make two assumptions in order to achieve model-independence. The first of these is the Effective Field Theory (EFT) assumption [21]. Here it is assumed that whatever particle mediates interactions between the SM and DM is massive enough that it can be integrated out of the theory, resulting in EFT contact interactions between the SM and DM. This allows one to enumerate all the possible interactions, the lowest dimensional of which are shown in Table 1. In general, each of the quark operators has 6 different $M_{*}$ values corresponding to the 6 quark flavors.

\begin{tabular}{|c|c|c|c|c|c|}
\hline Name & Operator & Coefficient & & & \\
\hline D1 & $\bar{\chi} \chi \bar{q} q$ & $m_{q} / M_{*}^{3}$ & & & \\
\hline D2 & $\bar{\chi} \gamma^{5} \chi \bar{q} q$ & $i m_{q} / M_{*}^{3}$ & Name & Operator & Coefficient \\
\hline D3 & $\bar{\chi} \chi \bar{q} \gamma^{5} q$ & $i m_{q} / M_{*}^{3}$ & $\mathrm{C} 1$ & $\chi^{\dagger} \chi \bar{q} q$ & $m_{q} / M_{*}^{2}$ \\
\hline D4 & $\bar{\chi} \gamma^{5} \chi \bar{q} \gamma^{5} q$ & $m_{q} / M_{*}^{3}$ & $\mathrm{C} 2$ & $\chi^{\dagger} \chi \bar{q} \gamma^{5} q$ & $i m_{q} / M_{*}^{2}$ \\
\hline D5 & $\bar{\chi} \gamma^{\mu} \chi \bar{q} \gamma_{\mu} q$ & $1 / M_{*}^{2}$ & $\mathrm{C} 3$ & $\chi^{\dagger} \partial_{\mu} \chi \bar{q} \gamma^{\mu} q$ & $1 / M_{*}^{2}$ \\
\hline D6 & $\bar{\chi} \gamma^{\mu} \gamma^{5} \chi \bar{q} \gamma_{\mu} q$ & $1 / M_{*}^{2}$ & $\mathrm{C} 4$ & $\chi^{\dagger} \partial_{\mu} \chi \bar{q} \gamma^{\mu} \gamma^{5} q$ & $1 / M_{*}^{2}$ \\
\hline D7 & $\bar{\chi} \gamma^{\mu} \chi \bar{q} \gamma_{\mu} \gamma^{5} q$ & $1 / M_{*}^{2}$ & $\mathrm{C} 5$ & $\chi^{\dagger} \chi G_{\mu \nu} G^{\mu \nu}$ & $\alpha_{s} / 4 M_{*}^{2}$ \\
\hline D8 & $\bar{\chi} \gamma^{\mu} \gamma^{5} \chi \bar{q} \gamma_{\mu} \gamma^{5} q$ & $1 / M_{*}^{2}$ & C6 & $\chi^{\dagger} \chi G_{\mu \nu} \tilde{G}^{\mu \nu}$ & $i \alpha_{s} / 4 M_{*}^{2}$ \\
\hline D9 & $\bar{\chi} \sigma^{\mu v} \chi \bar{q} \sigma_{\mu \nu} q$ & $1 / M_{*}^{2}$ & $\mathrm{R} 1$ & $\chi^{2} \bar{q} q$ & $m_{q} / 2 M_{*}^{2}$ \\
\hline $\mathrm{D} 10$ & $\bar{\chi} \sigma^{\mu v} \gamma^{5} \chi \bar{q} \sigma_{\mu \nu} q$ & $i / M_{*}^{2}$ & $\mathrm{R} 2$ & $\chi^{2} \bar{q} \gamma^{5} q$ & $i m_{q} / 2 M_{*}^{2}$ \\
\hline $\mathrm{D} 11$ & $\bar{\chi} \chi G_{\mu \nu} G^{\mu \nu}$ & $\alpha_{s} / 4 M_{*}^{3}$ & R3 & $\chi^{2} G_{\mu \nu} G^{\mu \nu}$ & $\alpha_{s} / 8 M_{*}^{2}$ \\
\hline $\mathrm{D} 12$ & $\bar{\chi} \gamma^{5} \chi G_{\mu \nu} G^{\mu \nu}$ & $i \alpha_{s} / 4 M_{*}^{3}$ & $\mathrm{R} 4$ & $\chi^{2} G_{\mu \nu} \tilde{G}^{\mu \nu}$ & $i \alpha_{s} / 8 M_{*}^{2}$ \\
\hline D13 & $\bar{\chi} \chi G_{\mu \nu} \tilde{G}^{\mu \nu}$ & $i \alpha_{s} / 4 M_{*}^{3}$ & & & \\
\hline $\mathrm{D} 14$ & $\bar{\chi} \gamma^{5} \chi G_{\mu \nu} \tilde{G}^{\mu \nu}$ & $\alpha_{s} / 4 M_{*}^{3}$ & & & \\
\hline
\end{tabular}

Table 1: The lowest dimensional EFT operators for interactions between quarks and gluons with dark matter. Dirac fermion DM is shown on the left, and real or complex scalar DM is shown on the right. D8 and D9 contribute to spin-dependent interactions and D1, D5, D11, C1, C3, C5, R1, and R3 contribution to spin-independent interactions. After applying MFV each quark operator picks up a sum over the three quark generations.. In general, there are two different $M_{*}$ values for each of these interactions corresponding to up and down type quarks. [21]

The second assumption is known as Minimal Flavor Violation (MFV) [22]. This is a purely observationally motivated concept stemming from the fact that precision flavor experiments have set a lower bound on the energy scale of generic new physics of $\Lambda_{N P}>O\left(10^{4}\right) \mathrm{TeV}$. This conflicts with the theoretical prediction of $O(1) \mathrm{TeV}$ for the appearance of physics beyond the $\mathrm{SM}$, and is also much too high to be probed at the LHC. The only way to reconcile these is to restrict the flavor structure of new physics at the TeV scale. MFV is one possible restriction that brings the experimental bound down to $O(1) \mathrm{TeV}$ as expected. The $\mathrm{SM}$ has a global $U_{q}(3) \times U_{u}(3) \times U_{d}(3)$ flavor symmetry in the quark sector, that is broken explicitly by the two quark Yukawa matrices. MFV makes the assumption that new physics, at least at the TeV scale, only breaks this global symmetry using the same Yukawa matrices. This immediately reduces the number of quark operators in Figure 1 by a factor of 3 and introduces a 
sum over the 3 generations. Because the Yukawa matrices are the only way to break flavor symmetry, each operator gains a specific dependence on the quark masses. Within this EFT/MFV framework, different types of WIMP searches can be easily compared in a model-independent way.

\subsection{Collider Signatures}

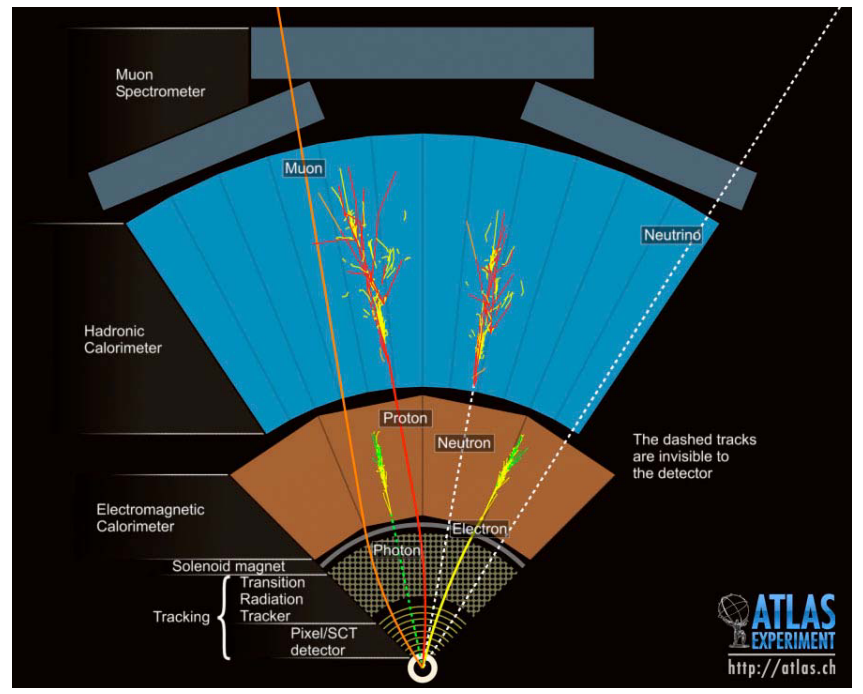

Figure 1: An illustration of how the different SM particles are detected by ATLAS. The inner detector measures the transverse momentum of any charged particles as they pass through a solenoidal magnetic field. The electromagnetic calorimeter measures the energy deposits of electromagnetically interacting particles, fully stopping electrons and photons. The hadronic calorimeter stops and measures energy deposits from any hadrons that escape the electromagnetic calorimeter. The only two particles able to easily escape the hadronic calorimeter are muons and neutrinos. The muon spectrometer makes a final momentum measurement of muons passing through a toroidal magnetic field, and neutrinos show up as missing transverse momentum. Although the longitudinal momentum of any collision is unknown, the transverse momentum is always 0 . Since momentum is conserved in any direction, any net transverse momentum in an event suggests the existence of invisible products in the opposite direction.

The ATLAS detector is designed as a general purpose detector that can search for any signs of new physics beyond the SM. It is composed of layered, cylindrical subdetectors which each serve to measure different types of particles. These subdetectors are laid out so that all known particles create unique signatures within the detector, as illustrated in Figure 1. WIMP dark matter, by definition, would not be detected directly by ATLAS. Instead, like neutrinos, it would show up as missing transverse momentum $\left(E_{\mathrm{T}}^{\mathrm{miss}}\right)$.

Because dark matter would be indistinguishable from neutrinos, known interactions that produce neutrinos will be a background for any collider search. This "real" source of $E_{\mathrm{T}}^{\mathrm{miss}}$ from neutrino production can occur in a number of processes, the primary ones being $Z \rightarrow v v, W \rightarrow \ell v$, and heavy quark decays. A typical analysis will have an irreducible background described by replacing the dark matter production with $Z \rightarrow v v$. There are also "fake" sources of $E_{\mathrm{T}}^{\text {miss }}$, which are due to instrumental effects. Because ATLAS does not have perfect acceptance or resolution, particles created in collisions can be mismeasured or not detected at all. $E_{\mathrm{T}}^{\text {miss }}$ is calculated from the vector sum of the $p_{\mathrm{T}}$ for all measured particles, so that any incorrect measurements will result in $E_{\mathrm{T}}^{\mathrm{miss}}$. Of course, ATLAS 
does have very good acceptance and resolution, so these events are rare. However, because of the huge number of collisions that occur, sources of fake $E_{\mathrm{T}}^{\text {miss }}$ can become a significant background to many dark matter searches. The best way of reducing this background is to remove events where reconstructed objects are aligned with the $E_{\mathrm{T}}^{\text {miss }}$ vector.

\subsection{Results}

In ATLAS, the model-independent WIMP searches are denoted "Mono-X", with X being some SM particle (e.g. W, Z, Higgs, jet). Because dark matter is not directly detectable, if there are no SM particles created in a collision there will be nothing to trigger on. The event will look identical to a non-collision event, and will not be recorded. The SM X particle is usually just initial state radiation (ISR) used to "tag" the events in order to make them detectable. Some searches make more use of $\mathrm{X}$ though, by looking at interactions that actually require the production of $\mathrm{X}$ in association with WIMPs. There are many more WIMP searches than the two I will present here, but these are representative of the different ATLAS dark matter analyses.

\subsubsection{Hadronic Mono-W/Z}

The Mono- $W / Z$ search looks for a high energy $W$ or $Z$ boson produced as ISR in WIMP production that then decays hadronically. Because the boson in the signal interaction will be boosted, it will decay into 2 collimated jets that can be reconstructed as a "fat jet". This fat jet is required to have a $p_{\mathrm{T}}>250 \mathrm{GeV}$ and a mass consistent with the $W$ or $Z$ masses, $50 \mathrm{GeV}<M_{\text {jet }}<120 \mathrm{GeV}$. The search is done in two inclusive signal regions, differing only in the cut on $E_{\mathrm{T}}^{\mathrm{miss}}$. The first region has $E_{\mathrm{T}}^{\text {miss }}>350 \mathrm{GeV}$ and the second has $E_{\mathrm{T}}^{\text {miss }}>500 \mathrm{GeV}$. Data is compared to background estimates within these two regions in order to place limits on a number of EFT operators for WIMP production. The data and background estimates are given in Table 2, and the $90 \%$ confidence level limits on $M_{*}$ are shown in Figure 2.

\begin{tabular}{c|c|c}
\hline \hline Process & $E_{\mathrm{T}}^{\text {miss }}>350 \mathrm{GeV}$ & $E_{\mathrm{T}}^{\text {miss }}>500 \mathrm{GeV}$ \\
\hline$Z \rightarrow v \bar{v}$ & $402_{-34}^{+39}$ & $54_{-10}^{+8}$ \\
$W \rightarrow \ell^{ \pm} v, Z \rightarrow \ell^{ \pm} \ell^{\mp}$ & $210_{-18}^{+20}$ & $22_{-5}^{+4}$ \\
$W W, W Z, Z Z$ & $57_{-8}^{+11}$ & $9.1_{-1.1}^{+1.3}$ \\
$t \bar{t}$, single $t$ & $39_{-4}^{+10}$ & $3.7_{-1.3}^{+1.7}$ \\
\hline Total & $707_{-38}^{+48}$ & $89_{-12}^{+9}$ \\
\hline Data & 705 & 89 \\
\hline \hline
\end{tabular}

Table 2: The final selection yields in the two signal regions. [23]

Like in any analysis, one of the most important aspects is background estimation. The irreducible backgrounds to this analysis are diboson production $W Z$ and $Z Z$, where a $Z$ decays to neutrinos and the second boson decays hadronically. Because of its very small cross-section though, the more frequent processes $Z \rightarrow v v$ and $W \rightarrow \ell v$ (where the charged lepton fails to be identified or a $\tau$ decays hadronically) dominate the background. These primary backgrounds are estimated using data-driven methods, while diboson and top backgrounds are estimated using Monte Carlo simulation. In order to reduce events with neutrinos from weak decays, a veto is placed on isolated leptons and photons. 


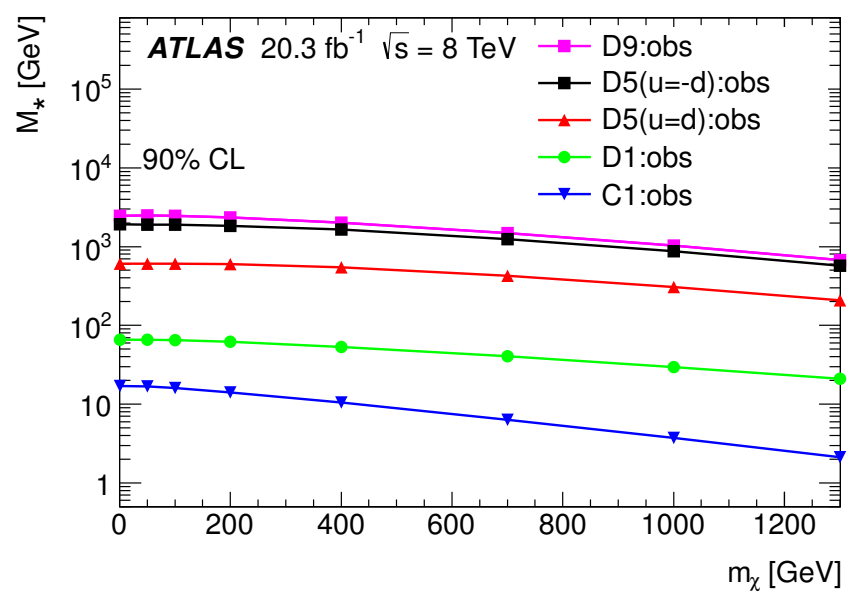

Figure 2: The limits set on $M_{*}$ for 4 of the EFT operators in the Mono- $W / Z$ search. Most of these assume universal couplings for up and down type quarks. However, for the D5 operator this channel sees a significant boost in sensitivity over mono-jet if the couplings for up and down type quarks have opposite sign. [23]

To eliminate sources of fake $E_{\mathrm{T}}^{\text {miss }}$ due to misreconstruction of jets, a veto is also placed on narrow jets closely aligned with the $E_{\mathrm{T}}^{\text {miss }}$ vector. The final background rejection cut reduces the high jet multiplicity backgrounds (top quark production) by rejecting any event with more than 1 narrow jet outside of the fat jet.

The final results agree very well with the SM expectation, and strong limits were placed on the operators C1, D1, D5, D9. As in most analyses, the couplings for up and down type quark interactions are assumed to be identical. As mentioned above though, this assumption is not required by MFV and can be relaxed. Here, if up and down type quarks have opposite sign couplings there is an interference effect between the two. The Mono-jet signal for D5 gets suppressed and the Mono- $W / Z$ signal gets enhanced. Limits in this special case are set as well.

\subsubsection{Leptonic Mono-Z}

The Mono- $Z$ search, on the surface, has a very similar signal to the Mono- $W / Z$ search above. The primary difference is that only $Z$ bosons are considered, and they are required to decay leptonically into 2 electrons or muons. Also, instead of only looking for ISR of the $Z$, this search also looks at additonal EFT operators that describe contact interactions directly between the $Z$ and WIMPs. The leptonic decay of the $Z$ requires the reconstruction of two leptons consistent with a $Z$ decay. This is defined as either 2 electrons or 2 muons with opposite signed charge, and an invariant mass $\left|m_{\ell \ell}-m_{Z}\right|<15 \mathrm{GeV}$. The remaining events are split into 4 inclusive signal regions corresponding to lower bounds on $E_{\mathrm{T}}^{\text {miss }}$ of $150,250,350$ and $450 \mathrm{GeV}$. Data is compared to background estimates within these 4 regions in order to place limits on a number of EFT operators for WIMP production. The data and background estimates are given in Table 3, and the $90 \%$ confidence level limits on $M_{*}$ are shown in Figure 3.

Because the topology of this signal is very different than for Mono- $W / Z$, the relevant backgrounds are also very different. Unlike before, the irreducible diboson production $Z Z$ is the largest background. $W Z$ is the second largest background, when the charged lepton is misidentified. Both of these backgrounds are estimated using Monte Carlo simulations. The remaining backgrounds $W W$, top, 


\begin{tabular}{c|c|c|c|c}
\hline \hline \multirow{2}{*}{ Process } & \multicolumn{4}{|c}{$E_{\mathrm{T}}^{\text {miss }}$ threshold [GeV] } \\
& 150 & 250 & 350 & 450 \\
\hline$Z Z$ & $41 \pm 15$ & $6.4 \pm 2.4$ & $1.3 \pm 0.5$ & $0.3 \pm 0.1$ \\
$W Z$ & $8.0 \pm 3.1$ & $0.8 \pm 0.4$ & $0.2 \pm 0.1$ & $0.1 \pm 0.1$ \\
$W W, t \overline{,}, Z \rightarrow \tau^{+} \tau^{-}$ & $1.9 \pm 1.4$ & $0_{-0.0}^{+0.7}$ & $0_{-0.0}^{+0.7}$ & $0_{-0.0}^{+0.7}$ \\
$Z+$ jets & $0.1 \pm 0.1$ & - & - & - \\
$W+$ jets & $0.5 \pm 0.3$ & - & - & - \\
\hline Total & $52 \pm 18$ & $7.2 \pm 2.8$ & 1.40 .9 & $0.4_{-0.4}^{+0.7}$ \\
\hline Data & 45 & 3 & 0 & 0 \\
\hline \hline
\end{tabular}

Table 3: The final selection yields in the two signal regions. [24]

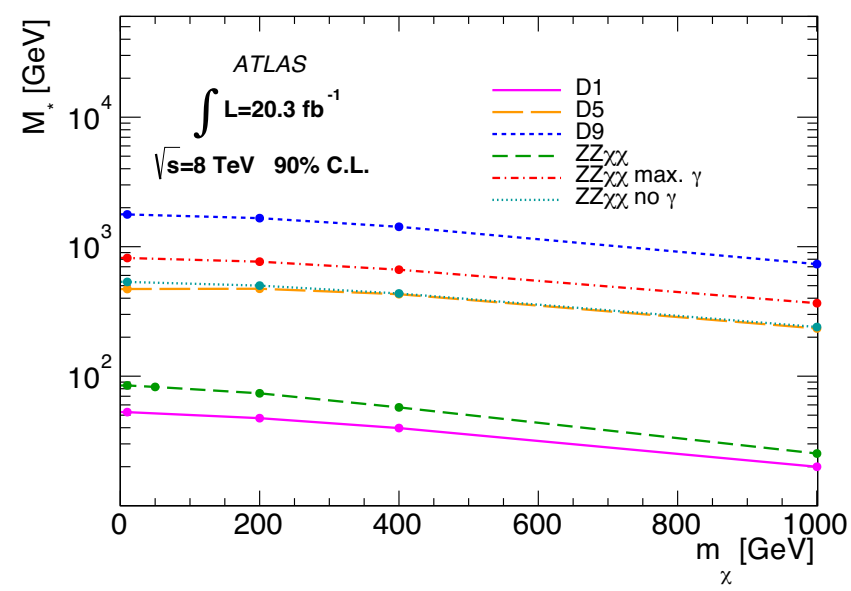

Figure 3: The limits set on $M_{*}$ for 5 of the EFT operators in the Mono- $/ Z$ search. For the dimension-7 operator coupling the $\mathrm{Z}$ to DM, two examples are shown corresponding to negligible and maximal contributions from $\gamma^{*}$. [24]

and single boson production all have very little contribution to the signal regions, because they are easily removed, and are estimated using a variety of data-driven methods. Three additional cuts are made to reduce all of these backgrounds. The first is a veto on any additional high $p_{\mathrm{T}}$ jets or leptons, which removes a large portion of the diboson and top backgrounds. The second is a veto on events where the reconstructed $Z$ boson is aligned with the $E_{\mathrm{T}}^{\text {miss }}$, to eliminate sources of fake $E_{\mathrm{T}}^{\mathrm{miss}}$ from misreconstructed leptons. The final cut is to require $\left|p_{\mathrm{T}}(Z)-E_{\mathrm{T}}^{\mathrm{miss}}\right| / p_{\mathrm{T}}(Z)<0.5$. This ensures that the event is consistent with a $Z$ boson recoiling off something invisible.

As before, the results agree very well with the expected SM background within errors. This allows limits to be replaced on $M_{*}$ for each of the EFT operators. Although the Mono- $W / Z$ search provides stronger limits on the quark couplings, new limits have been set on EFT operators that couple the $Z$ boson directly to dark matter. 


\subsubsection{Summary}

These two analyses, and others, were able to place limits on a number of the EFT operators mentioned under the MFV condition. The searches are model-independent, but do make two assumptions that need to be considered. MFV is a sufficient but not necessary condition to get $O(1) \mathrm{TeV}$ scale new physics. This needs to be kept in mind when interpreting these results, because they only apply to models which fit into the MFV framework. The justification for using an EFT model was that the mediator fields are massive enough to be integrated out. This condition is difficult to define formally, as $M_{*}$ is a function of the mediator mass and its couplings, which are free model-dependent parameters in general. However, it is a reasonable requirement that each coupling be below $\sqrt{4 \pi}$, in order for the theory to be perturbative. In this case a validity constraint can be set as $Q_{t r}<M_{m e d}$, where $Q_{t r}$ is the momentum transferred to the WIMPs and $M_{m e d}$ is the minimum mediator mass allowed for any given $M_{*}$ under the condition of perturbativity [25]. This is not a strict cutoff, but when it is satisfied the model-independent EFT approach is definitely justified. If it is not satisfied, the results can either underestimate or overestimate the true limits, depending on what model is used, and the EFT approach loses its reliability.

Using the methods described in [21], the expected WIMP-nucleon cross-section of each EFT operator can be calculated as a function of $M_{*}$ and the WIMP mass. The limits set on $M_{*}$ for each of the operators can then be translated into limits on the WIMP-nucleon cross-section and compared directly to other WIMP searches. The results of this process are shown in Figure 4 for both spindependent and spin-independent interactions.
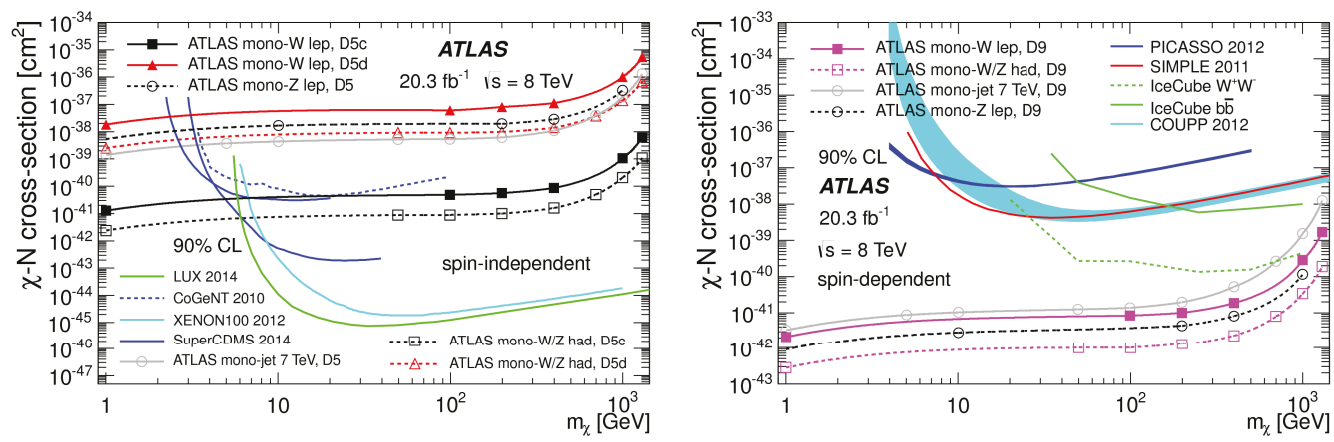

Figure 4: The WIMP-nucleon cross-section limits placed by a number of experiments. The direct detection searches include LUX, CoGeNT, XENON100, SuperCDMS, PICASSO, SIMPLE and COUPP. IceCube is an indirect detection search that has also placed limits on spin-dependent interactions. The four ATLAS searches are the two discussed here, and the leptonic mono- $W$ search, the mono-jet $7 \mathrm{TeV}$ search. [26]

\section{Large Extra Dimension Searches}

\subsection{Background}

One of the main appeals of adding extra dimensions to our $3+1$ dimensional spacetime comes from the hierarchy problem. According to our current theories, the electroweak force is $O\left(10^{32}\right)$ times stronger than gravity. A priori, there is no reason to expect such a large gap between the dimensionless coupling constants that define these two scales. The Standard Model is, for the most part, in the 
$\sim \mathrm{MeV}-\mathrm{TeV}$ range, while the Planck scale is all the way at $O\left(10^{15}\right) \mathrm{TeV}$. So far, there is no known explanation why nature is so finely tuned with gravity being so much weaker than the other forces (equivalently, the Higgs mass being so much smaller than the Planck mass). One proposed solution is ADD theory [27].

ADD theory is a fairly simple model where $d$ flat compactified spatial dimensions, each of size $R$, are introduced. The SM remains confined to the ordinary $3+1$ dimensional spacetime, while gravity is allowed to propagate in all of them. This has the effect of weakening gravity at distances smaller than $R$ and introduces a new parameter $M_{D}$, the fundamental gravitational scale. At distances much less than $R$, gravitational forces are determined by $M_{D}$ rather than $M_{\text {Planck }}$ and the force law is $O\left(r^{-(2+d)}\right)$. The relation between the observed constant at large distances and fundamental scale is given by $M_{\text {Planck }}^{2} \sim M_{D}^{d+2} R^{d}$.

Gravity has only been tested down to the millimeter scale, so that as long as $d>1, M_{D}$ can be brought down to the $\mathrm{TeV}$ scale without any conflict from experimental data. If the true gravitational scale is low enough to be probed by the LHC, it would cause some very unique phenomena. First, gravitational standing waves, known as Kaluza Klein (KK) towers, would form in each of the $d$ compact dimensions. There would be an infinite number of them with masses related by $M_{n} \sim n R^{-1}$, where $n \geq 1$. Additionally, black hole (BH) formation would become possible during LHC collisions. There are two different types of black holes that may form, distinguished by a second parameter $M_{t h}$. $M_{t h}$ is the minimum mass a black hole must have for the semi-classical approximation to hold. Put another way, black holes with masses above $M_{t h}$ will decay thermally through Hawking radiation. Black holes with masses below this threshold are known as quantum black holes (QBH), and require a theory of quantum gravity to describe. There are many searches at ATLAS for QBHs [28][29], but they will not be discussed here.

\subsection{Collider Signatures}

KK towers and BHs are predicted to have very unique, but non-resonant signatures. A black hole decaying thermally will necessarily have a very high mass, because there are tight bounds on how low $M_{D}$ can be and $M_{t h}>>M_{D}$ by definition. If one is produced, it should decay isotropically into a very large number of particles. Although charge, energy, and angular momentum are conserved in black hole decay, certain symmetries of the SM may not be (e.g. baryon or lepton number symmetry). These three traits allow a search for BH production to be done with very little SM background.

KK towers are completely different phenomenon. While their masses can vary, the mass splitting is based solely on the parameter $R$. At $R \sim 1 \mu \mathrm{m}$, a typical value for the LHC energy scale, the mass splitting is $\Delta M \sim 10^{-10} \mathrm{GeV}$. So while KK tower interactions would in principle be resonant, these resonances would be smeared out into a smoother spectrum. Gravitons and KK towers, in general, would be invisible at ATLAS, but Drell-Yan (DY) production of SM particles through virtual KK mediators could be observed. Because of the fine mass splitting, this would create a smeared out DY spectrum that would behave very differently at high masses than any known SM process.

\subsection{Results}

There are many different searches at ATLAS for large extra dimensions. These typically fall into 3 categories: Thermal BHs, QBHs, and KK towers. QBH display resonant behavior and are discussed in a separate talk. For the other two, I will present 1 example of each. 


\subsubsection{Thermal Black Holes}

This particular $\mathrm{BH}$ search looks for high $p_{\mathrm{T}}$ leptons and jets in very "energetic" events. The discriminating variable used is $\sum p_{\mathrm{T}}$, which is the scalar sum of the $p_{\mathrm{T}}$ of each lepton or jet with $p_{\mathrm{T}}>60 \mathrm{GeV}$. The signal selection requires at least 3 objects (electrons, muons, or jets) with $p_{\mathrm{T}}>$ $100 \mathrm{GeV}$, where at least one of them is a lepton. This is then split into 7 inclusive signal regions with cuts on $\sum p_{\mathrm{T}}$ ranging from $2,000 \mathrm{GeV}$ to $3,200 \mathrm{GeV}$ in $200 \mathrm{GeV}$ increments. The data is further split into electron and muon channels, where the highest $p_{\mathrm{T}}$ lepton determines which channel each event is in. Data is compared to the SM background estimate within these 14 regions in order to place limits on $M_{t h}$ as a function of $M_{D}$. Because this is a search for semi-classical black holes, limits can not be placed directly on $M_{D}$ without a quantum gravity theory that would be able to predict the value of $M_{t h}$. The observed data and estimated backgrounds in the signal regions are shown in Table 4 and the final $\sum p_{\mathrm{T}}$ distributions are shown in Figure 5.

\begin{tabular}{|c||c|c||c|c|}
\hline \multicolumn{1}{|c||}{} & \multicolumn{2}{c||}{ Electron Channel } & \multicolumn{2}{c|}{ Muon Channel } \\
Min. $\sum p_{\mathrm{T}}[\mathrm{GeV}]$ & Expected Background & Data & Expected Background & Data \\
\hline 2000 & $44 \pm 12$ & 47 & $22.8 \pm 5.4$ & 27 \\
\hline 2200 & $19 \pm 7$ & 22 & $10.1 \pm 3.2$ & 12 \\
\hline 2400 & $8.2 \pm 3.7$ & 5 & $4.5 \pm 1.9$ & 7 \\
\hline 2600 & $3.5 \pm 2.1$ & 2 & $2.0 \pm 1.3$ & 2 \\
\hline 2800 & $1.5 \pm 1.2$ & 0 & $0.89 \pm 0.82$ & 2 \\
\hline 3000 & $0.65_{-0.65}^{+0.69}$ & 0 & $0.40_{-0.40}^{+0.53}$ & 0 \\
\hline 3200 & $0.28_{-0.28}^{+0.40}$ & 0 & $0.18_{-0.18}^{+0.34}$ & 0 \\
\hline
\end{tabular}

Table 4: The final selection yields in the 14 signal regions. [30]

No additional cuts are made to reject SM backgrounds, as the SM contribution to the high $\sum p_{\mathrm{T}}$ region is already sufficiently small. The largest background is $W+$ jets, and is estimated the same as every other significant SM background. Monte Carlo simulations are first normalized to data in a control region where $700 \mathrm{GeV}<\sum p_{\mathrm{T}}<1500 \mathrm{GeV}$. Because even the simulations have very poor statistics in the signal region, these need to be extrapolated. To do this, an ansatz function is defined and then fit to each background in the region $\sum p_{\mathrm{T}}>700 \mathrm{GeV}$. This fit is then used as the final background estimate. The resulting SM estimate agrees very well with the observed data, allowing limits to be set on the ADD parameters.

\subsubsection{Kaluza-Klein Towers}

The KK tower search looks for the DY signature, mentioned above, in the production of dilepton pairs. The signal region is very simple and is defined by two cuts. The first is that at least one same flavor, opposite sign lepton pair exists in the event. The second is that the invariant mass $\left(m_{\ell \ell}\right)$ of the highest $\sum p_{\mathrm{T}}$ pair of leptons is above $1.9 \mathrm{TeV}$. No data events are observed within the signal region, which is consistent with the SM estimate. The final selection yields are given in Table 5 , and the $m_{\ell \ell}$ distributions are shown in Figure 6.

Although the main irreducible background is the $Z / \gamma^{*}$ Drell-Yan production of dilepton pairs, at high $m_{\ell \ell}$ none of the SM backgrounds contribute significantly. The multijet and $W+$ jets back- 

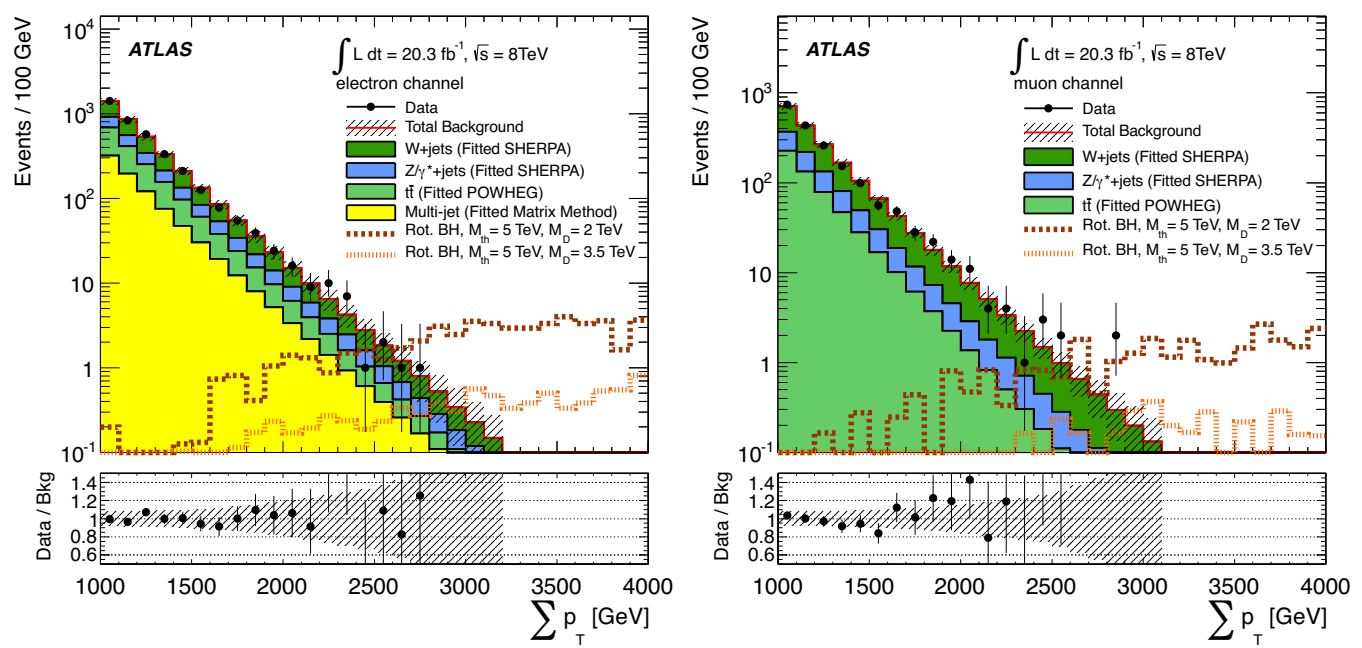

Figure 5: The $\Sigma p_{\mathrm{T}}$ distributions in the electron and muon channels. In addition to data and MC, two possible signals that are ruled out by the analysis have been plotted.[30]

\begin{tabular}{c|c|c}
\hline \hline \multirow{2}{*}{ Process } & $m_{e e}[\mathrm{GeV}]$ & $m_{\mu \mu}[\mathrm{GeV}]$ \\
\cline { 2 - 3 } & $1900-4500$ & $1900-4500$ \\
\hline Drell-Yan & $0.43 \pm 0.12$ & $0.44 \pm 0.09$ \\
Top quarks & $<0.002$ & $<0.006$ \\
Multi-Jet \& $W+\mathrm{Jets}$ & $0.062 \pm 0.005$ & $<0.001$ \\
Diboson & $0.053 \pm 0.005$ & $0.047 \pm 0.005$ \\
Photon-Induced & $0.06 \pm 0.06$ & $0.05 \pm 0.05$ \\
\hline Total SM & $0.61 \pm 0.13$ & $0.54 \pm 0.09$ \\
\hline Data & 0 & 0 \\
\hline $\mathrm{SM}+\mathrm{ADD}\left(M_{s}=3.5 \mathrm{TeV}\right)$ & $21.6 \pm 2.1$ & $12.2 \pm 1.4$ \\
$\mathrm{SM}+\mathrm{ADD}\left(M_{s}=4.75 \mathrm{TeV}\right)$ & $2.95 \pm 0.28$ & $1.90 \pm 0.20$ \\
\hline \hline
\end{tabular}

Table 5: The final selection yields in the two signal regions. [31]

grounds are estimated using data-driven methods, while Monte Carlo simulations are used for the other backgrounds. The entire SM estimate is normalized to data in the region around the $Z$ mass $80 \mathrm{GeV}<m_{\ell \ell}<120 \mathrm{GeV}$. The resulting SM estimate agrees very well with the observed data for the entire $m_{\ell \ell}$ range, allowing limits to be set directly on the string scale $M_{S}$, which is used as the ultraviolet cutoff and is directly related to $M_{D}$. 

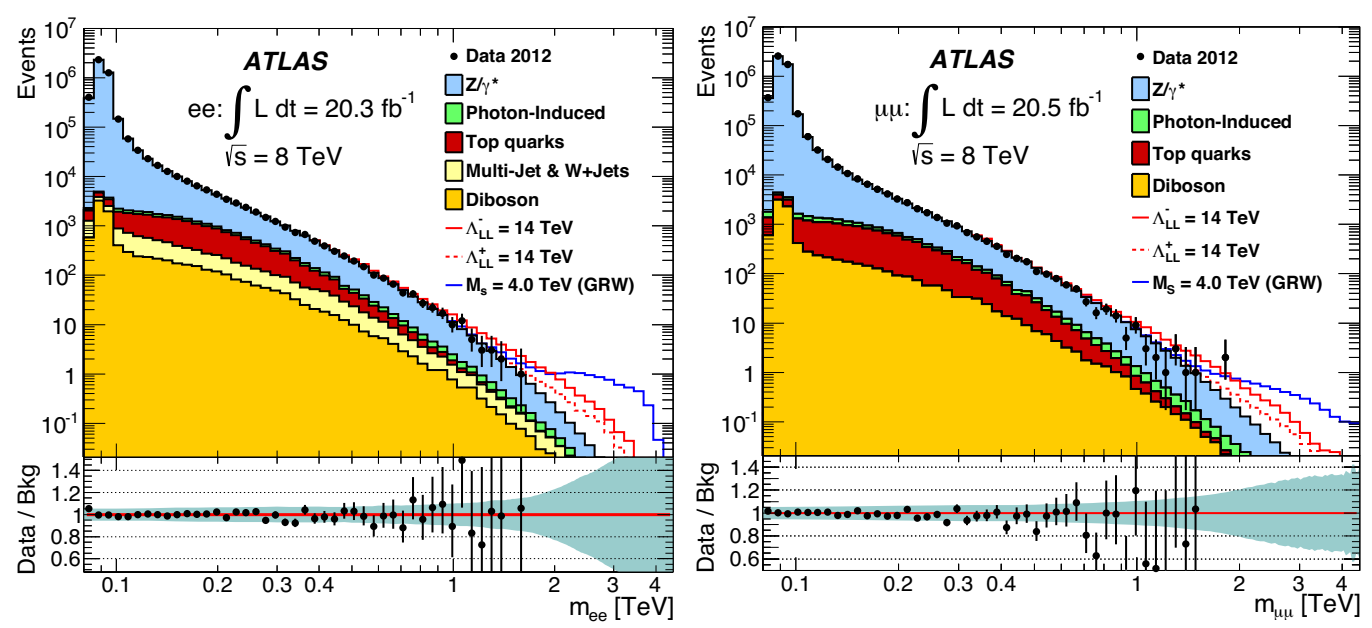

Figure 6: The $m_{\ell \ell}$ distributions out to $4.5 \mathrm{TeV}$. Shown are each SM background estimate, and one example signal corresponding to $M_{S}=4.0 \mathrm{TeV}$. [31]

\subsection{Summary}

These two analyses were able to place very strong limits on the parameters of ADD theory. Examples of these limits are shown in Figure 7. Both of these analyses searched beyond just ADD theory and considered a third parameter $M_{S}$, the string scale. The thermal black hole search can be reinterpreted as a search for string ball creation, and the KK tower search actually only places limits on $M_{S}$. However, the assumption made in the analysis is that $M_{S}$ is a simple function of $M_{D}$ given by Equation 1, so that the limits can be rewritten in terms of $M_{D}$. This ends up being about $M_{D} \gtrsim 1 \mathrm{TeV}$, depending on the particular model used. So without going into details of $M_{S}$, both analyses are able to place limits on $M_{D}$ and $M_{t h}$.

$$
M_{S}=2 \sqrt{\pi}\left[\Gamma\left(\frac{n}{2}\right)\right]^{1 /(n+2)} M_{D}
$$

\section{Conclusion}

ATLAS has been able to search for phenomena typically associated with cosmological scales such as black holes, string balls, dark matter, and large extra dimensions. The limits placed on low mass $\mathrm{DM}\left(m_{D M} \lesssim 10 \mathrm{GeV}\right)$ are the strongest to date. By using EFT and MFV, these can be combined with results from direct and indirect detection to provide strong limits over a very wide mass range for spin-independent interactions. For spin-dependent interactions, the collider limits are stronger than any other search done. Additionally, there are many operators inaccessible to other DM searches that have been probed by ATLAS. Finally, the fundamental gravitational scale $M_{D}$ has been tested well into the TeV scale, pushing the bounds of ADD theory.

Run II, scheduled to begin in 2015 will start colliding protons at a center of mass energy of $13 \mathrm{TeV}$, making a much higher energy scale accessible to these searches. Over the 2 year run time about $100 \mathrm{fb}^{-1}$ is expected to be delivered to ATLAS, over 4 times the data collected from Run I. This higher energy and higher luminosity will significantly strengthen all of the limits set above, and hopefully discover some sign of physics beyond the Standard Model. 

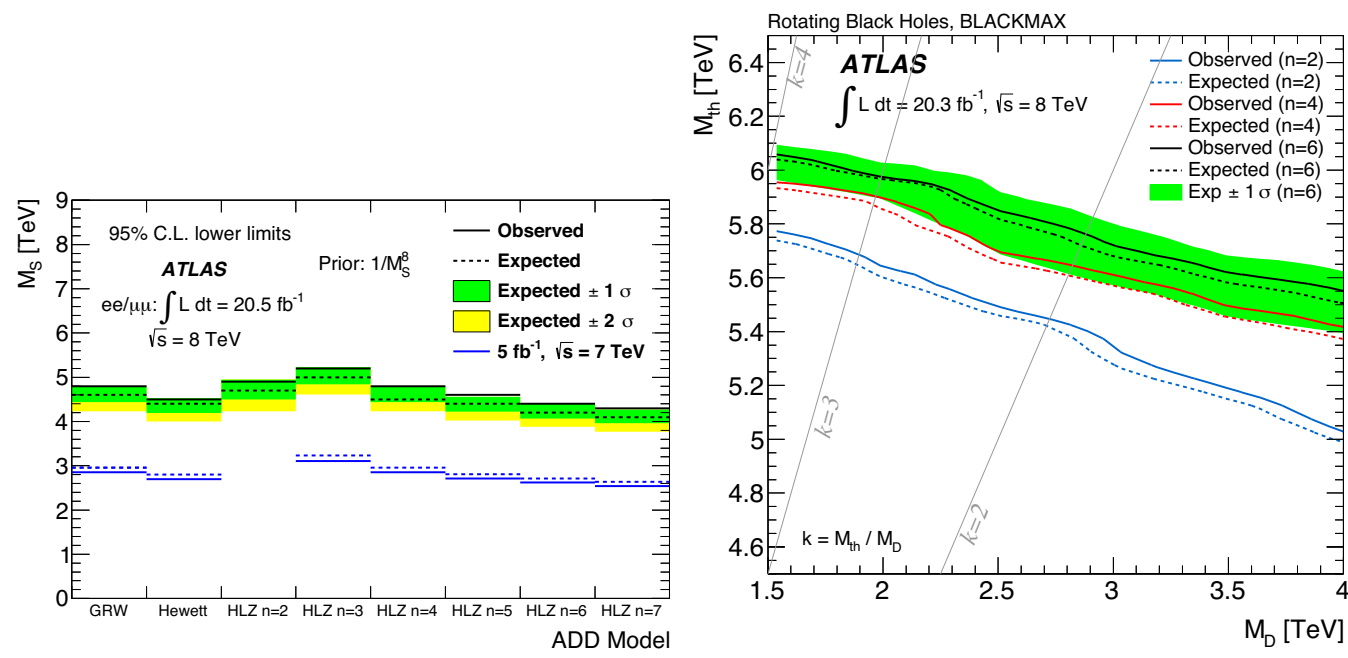

Figure 7: The final limits set on $M_{S}, M_{D}, M_{t h}$ by both ADD searches. On the left, the limits are set on $M_{S}$ which is a function of $M_{D}$. These are the strongest limits that have been placed on $M_{D}$. On the right are the limits set for 3 different values of $d$, for one specific BH model. Many different models are considered, but all give similar results.[31][30]

\section{References}

[1] F. Zwicky, Helv.Phys.Acta 6, 110 (1933)

[2] V.C. Rubin, J. Ford, W. Kent, Astrophys.J. 159, 379 (1970)

[3] D. Clowe, M. Bradac, A.H. Gonzalez, M. Markevitch, S.W. Randall et al., Astrophys.J. 648, L109 (2006), arXiv: astro-ph/0608407

[4] G.R. Blumenthal, S. Faber, J.R. Primack, M.J. Rees, Nature 311, 517 (1984)

[5] N. Jarosik, C.L. Bennett, J. Dunkley, B. Gold, M.R. Greason, M. Halpern, R.S. Hill, G. Hinshaw, A. Kogut, E. Komatsu et al., ApJS 192, 14 (2011), arXiv: 1001.4744

[6] R.A. Knop et al. (Supernova Cosmology Project), Astrophys.J. 598, 102 (2003), arXiv: astro-ph/0309368

[7] Phys. Rev. Lett. 113, 121101 (2014)

[8] O. Adriani, G. Barbarino, G. Bazilevskaya, R. Bellotti, M. Boezio et al., Phys.Rev.Lett. 102, 051101 (2009), arXiv:0810.4994

[9] W.B. Atwood, A.A. Abdo, M. Ackermann, W. Althouse, B. Anderson, M. Axelsson, L. Baldini, J. Ballet, D.L. Band, G. Barbiellini et al., ApJ 697, 1071 (2009), arXiv : 0902 . 1089

[10] M. Aartsen et al. (IceCube), Phys.Rev. D88, 122001 (2013), arXiv: 1307.3473

[11] R. Agnese et al. (CDMS), Phys. Rev. Lett. 111, 251301 (2013), arXiv: 1304 . 4279

[12] E. Aprile et al. (XENON100), Phys. Rev. Lett. 109, 181301 (2012), arXiv: 1207.5988

[13] D. Akerib et al. (LUX), Phys. Rev. Lett. 112, 091303 (2014), arXiv: 1310.8214

[14] G. Angloher et al. (CRESST-II) (2014), arXiv: 1407. 3146

[15] C.E. Aalseth, P.S. Barbeau, J. Colaresi, J.I. Collar, J. Diaz Leon, J.E. Fast, N.E. Fields, T.W. Hossbach, A. Knecht, M.S. Kos et al., Phys. Rev. D 88, 012002 (2013), arXiv: 1208.5737

[16] E. Behnke et al. (COUPP), Phys. Rev. D86, 052001 (2012), arXiv: 1204 . 3094 
[17] M. Felizardo, T.A. Girard, T. Morlat, A.C. Fernandes, A.R. Ramos, J.G. Marques, A. Kling, J. Puibasset, M. Auguste, D. Boyer et al., Phys. Rev. D 89, 072013 (2014)

[18] S. Archambault et al. (PICASSO), Phys. Lett. B711, 153 (2012), arXiv: 1202 . 1240

[19] ATLAS Collaboration, JINST 3, S08003 (2008)

[20] L. Evans, P. Bryant, JINST 3, S08001 (2008)

[21] J. Goodman, M. Ibe, A. Rajaraman, W. Shepherd, T.M. Tait et al., Phys.Rev. D82, 116010 (2010), arXiv: 1008.1783

[22] G. D'Ambrosio, G. Giudice, G. Isidori, A. Strumia, Nucl.Phys. B645, 155 (2002), arXiv: hep-ph/0207036

[23] ATLAS Collaboration, Phys.Rev.Lett. 112, 041802 (2014), arXiv: 1309.4017

[24] ATLAS Collaboration, Phys.Rev. D90, 012004 (2014), arXiv: 1404.0051

[25] G. Busoni, A. De Simone, E. Morgante, A. Riotto, Phys.Lett. B728, 412 (2014), arXiv: 1307.2253

[26] ATLAS Collaboration, JHEP 1409, 037 (2014), arXiv: 1407.7494

[27] N. Arkani-Hamed, S. Dimopoulos, G. Dvali, Phys.Lett. B429, 263 (1998), arXiv:hep-ph/9803315

[28] ATLAS Collaboration, Phys.Lett. B728, 562 (2014), arXiv: 1309.3230

[29] ATLAS Collaboration, Phys.Rev.Lett. 112, 091804 (2014), arXiv: 1311.2006

[30] ATLAS Collaboration, JHEP 1408, 103 (2014), arXiv: 1405.4254

[31] ATLAS Collaboration (2014), arXiv: 1407.2410 